\title{
Mobility edge and Black Hole Horizon
}

\author{
Alexander Gorsky ${ }^{1,2, *}$ \\ ${ }^{1}$ Institute of Information Transmission Problems of the Russian Academy of Sciences, Moscow, Russia, \\ ${ }^{2}$ Moscow Institute of Physics and Technology, Dolgoprudny 141700, Russia
}

\begin{abstract}
We conjecture that the mobility edge in the 4D Euclidean Dirac operator spectrum in QCD in the deconfined phase found in the lattice studies corresponds to the near black hole $(\mathrm{BH})$ horizon region in the holographic dual. We present some evidences both from the field theory side and from the worldsheet theory of long open string.
\end{abstract}

\section{Introduction}

In this talk I shall mainly follow the conjectures and questions formulated in [1]. First, let me explain the motivation of our study. The 4D Euclidean Dirac operator spectrum in QCD is the important observable both in the confined and deconfined phases. The famous Casher-Banks relation [2] for the chiral condensate in terms of the spectral density at the origin in the confined phase is the most immediate example. The interesting sum rules can be formulated for eigenvalues [3] and two types of matrix models provide the powerful tools for a investigation of the Dirac operator spectral properties (see [4] for review). One matrix model corresponds to the zero momentum sector of the Chiral Lagrangian while the second model mimics the fermion determinant integrated over the moduli space of a instanton-antiinstanton ensemble in QCD ground state.

It was suggested in [5] to attack the spectral problem for the Dirac operator by the tools familiar in solid state physics and treat the Euclidean Dirac operator in 4D as the Hamiltonian with respect to the additional fifth time coordinate identified with the Schwinger proper time. This viewpoint allowed to identify the pion as the analogue of the diffuson, relate the diffusion constant with the pion decay constant and identify the standard energy and time scales familiar in the disordered systems.

According to this logic the question concerning the localization of the eigenmodes of the Dirac operator is quite natural. The spectral formfactor and spectral correlator are standard and quite informative objects which allow to identify the localization properties of the eigenfunctions. The more involved quantity is the level spacing distribution which distinguishes the parts of the spectrum with localized and delocalized modes. The delocalized modes are subject of the level interaction and obey the Wigner-Dyson statistics while the localized modes do not correlate and obey the Poisson statistics. There can be the mobility edge separating the localized and delocalized modes in the $d \geq 3$ where $\mathrm{d}$ is dimension of space.

The localization properties of the Dirac operator spectrum in QCD have been investigated in the lattice version and it has been found a bit surprisingly that all modes are delocalized

\footnotetext{
*e-mail: shuragor@mail.ru
} 
in the confined phase while there is the mobility edge in the deconfined phase [6]. Low energy modes in the deconfined phase are localized while high energy part of the spectrum is delocalized. Moreover it has been found [7] that the mobility edge appears exactly at the temperature of the deconfinement phase transition.

In [1] we attack the problem of the Dirac operator spectrum from the holographic viewpoint. The first indication of the holographic picture behind the scene is the relation between the Schwinger proper time and the radial coordinate in the ADS space [8]. This relation still survives for the case of the external field [9] and the finite temperature [10]. On the other hand the dynamics in the radial direction amounts the holographic RG flows [11]. Hence the time conjugated to the 4D Dirac operator considered as the Hamiltonian in 4D Euclidean space is the RG time in holographic RG flows and many questions can be reformulated in RG terms.

The deconfinement transition in holographic QCD corresponds to the change of the bulk geometry which involves the thermal AdS at $T<T_{c}$ and AdS BH at $T>T_{c}[12,13]$. Hence it is natural to question how the emergence of $\mathrm{BH}$ in the deconfined phase and the mobility edge in the 4D Dirac operator spectrum are correlated. We have found the evidences that indeed the corresponding energy scales - radius of $\mathrm{BH}$ and the mobility edge are approximately the same. We shall use the field theory arguments as well as the arguments based on the worldsheet theory of a long open string extended along the radial coordinate in AdS which represents the matter in fundamental representation.

Let us emphasize from the very beginning that the localization phase transition in the Dirac operator spectrum in QCD which we discuss in this study is not the conventional Anderson-type transition. Indeed the phase transitions driven by disorder depend on the dimensionality and the dispersion relation for the degrees of freedom. It was observed long time ago in [14] that the disorder driven transition for the Dirac operator differs from the transition for the Schrodinger operator. The key difference concerns the role of spectral density as the order parameter. In the Anderson transition the spectral density does not play any essential role and only a spectral formfactor and higher spectral correlators matter. On the other hand for the disordered Dirac operator in higher dimensions the spectral density at the origin becomes the order parameter. Moreover it was argued recently [15] that the non-Anderson disorder driven phase transition takes place if

$$
d>2 \gamma, \quad E \propto k^{\gamma}
$$

where $\gamma$ defines the dispersion relation, $\gamma=1$ for the Dirac operator. The non-Anderson transition can be supplemented with the additional localization transition if it is allowed by the symmetry.

We shall be interested in the Dirac operator spectrum in $4 d$ Euclidean space hence indeed the non-Anderson transition can be expected. The phase transition with the chiral symmetry restoration results at $T=T_{c}$ to vanishing of spectral density at the origin while at higher temperatures in the deconfined phase the mobility edge indicating the localization transition appears.

\section{Dirac operator in QCD}

Let us recall some results concerning the Dirac operator spectrum. The partition function of QCD reads as

$$
Z_{Q C D}=\int d A_{\mu} \prod_{f=1}^{N_{f}} \operatorname{det}\left(i D+m_{f}\right) \exp \left(-S_{Y M}(A)\right)
$$


and can be decomposed into the sectors with fixed topological charges weighted with the $\theta$ term

$$
Z_{Q C D}(\theta)=\sum_{v} e^{i \theta v} Z_{v, Q C D}
$$

We shall deal with the eigenvalue equation for the 4D Euclidean Dirac operator

$$
\hat{D}(A) \psi_{n}=i \lambda_{n} \psi_{n}
$$

which coincides with the Dirac equation for the imaginary fermion mass $m=i \lambda$. The spectral density is defined as

$$
\rho(\lambda)=<\sum_{n} \delta\left(\lambda-\lambda_{n}\right)>_{Q C D}
$$

which at the origin in the confined phase yields the chiral condensate [2]

$$
<\bar{\Psi} \Psi>=\Sigma=\frac{\pi \rho(0)}{V}
$$

The spectral density can be derived from the discontinuity of the resolvent across the imaginary axis

$$
\Sigma(z)=<V^{-1} \operatorname{Tr} \frac{1}{D+z}>
$$

Let us summarize the results concerning the spectral correlators for Dirac operator in the confining phase [4]. In the bulk of the spectrum where $\lambda \propto O(1)$ at large $\mathrm{N}$ one has

$$
\begin{gathered}
R\left(x_{1}, x_{2}\right)=\operatorname{det}\left(K\left(x_{i}, x_{j}\right)\right)_{i, j=1,2} \\
K(x-y)=\frac{\sin \pi(x-y)}{\pi(x-y)}
\end{gathered}
$$

In the microscopic region near the origin the kernel gets modified to

$$
K(x-y)=\sqrt{x y} \frac{x J_{\alpha+1}(x) J_{\alpha}(y)-y J_{\alpha}(x) J_{\alpha+1}(y)}{x^{2}-y^{2}}
$$

where $J_{\alpha}$ denotes Bessel function and $\alpha=N_{f}+|v|$. The microscopic kernel is sensitive to the topological sector and the microscopic spectral density is related to the kernel $\rho_{s}(z)=K(z, z)$

$$
\rho_{s}(z)=\frac{z}{2}\left[J_{N_{f}+|v|}^{2}(z)-J_{N_{f}+|v|+1}(z) J_{N_{f}+|v|-1}(z)\right]
$$

This spectral density can be obtained from the partially quenched Chiral Lagrangian. The kernel in the microscopic region can be expressed in terms of the partition function with two additional flavors of imaginary masses $m_{1}, m_{2}$ [16]

$$
K(x, y)=\frac{1}{2}(x y)^{N_{f}+1 / 2} \frac{Z_{N_{f}+2}\left(m_{1}=i x, m_{2}=i y\right)}{Z_{N_{f}}}
$$

The lattice studies definitely demonstrate $[6,7]$ that all eigenvalues of $4 \mathrm{~d}$ Euclidean Dirac operator in the confined phase are delocalized hence it behaves as $4 \mathrm{~d}$ metal. The metallic property of the Dirac operator in confined phase of QCD is quite counterintuitive since quark is assumed to be confined in $3 \mathrm{~d}$ space. The most naive argument in favor of the metal phase is that at $T=0$ we have $O(4)$ symmetry in Euclidean space and expected delocalization in Euclidean time implies the delocalization in the $3 \mathrm{~d}$ space. However since Polyakov line vanishes in the confined phase there is no $O(4)$ symmetry breaking disorder. Another standard 
argument applied to the zero eigenvalue only is that the fermionic zero modes get delocalized in the instanton-antiinstanton ensemble. Argument of Parisi [17] claims that the eigenvalues of the Dirac operator in confined phase have to interact to provide the finite density at the origin and hence obey the Wigner-Dyson statistics.

Here we suggest that the topological delocalization phenomenon namely that the disorder driven localization transition is strongly influenced by the topological terms $[18,19]$ is relevant. If the topological terms are added to the action the two-couplings RG flow occurs and some universal phenomena get manifested. Namely at some quantized values of the chemical potentials for the topological terms the delocalized modes appear in the spectrum. There are examples of this phenomena at "' $\theta=\pi$ " in the QHE effect [20,21]. The tiny aspects of such regime have been recently reconsidered in 4D Yang-Mills theory [22]. Generically there is no mobility edge in disordered $(1+1)$ and $(2+1)$ systems however the topological delocalization phenomenon occurs at some values of the chemical potential for the winding number. It is the manifestation of the instanton effects in the two-couplings RG flows.

We conjecture that the similar topological delocalization phenomenon occurs in the $(4+1)$ case as well in the confined phase. The mass dependence due to the axial anomaly goes through $m^{N_{f}} e^{i \theta}$ hence to have imaginary masses as required for eigenvalues of the Dirac operator we can introduce the particular value of $\theta$ which depends on the number of flavors. In particular for $N_{f}=0$ when the Dirac operator plays the role of probe $\theta=\pi$ and generically $\theta=\frac{\pi\left(N_{f}+2\right)}{2}$. With this interpretation the delocalization of Dirac operator modes occurs only at the particularly values of $\theta$.

\section{Holographic deconfined QCD}

Turn to the holographic QCD and consider the Witten-Sakai-Sugimoto geometry [12, 23] It involves the $N_{c}$ D4 branes wrapped around the cylinder with the boundary conditions breaking SUSY. At large $N_{s}$ the D4 branes pinch the cylinder which turns into the cigar. The total 10d geometry looks as $R^{3,1} \times S^{4} \times(r, \phi)$ at small temperature. Let drop off all space-time and $S^{4}$ dependence of the fields we are interested in for a moment. Hence the relevant part of the holographic geometry is the cigar in $(r, \phi)$ coordinates

$$
d s^{2}=(r / R)^{3 / 2} f(r) d \phi^{2}+(R / r)^{3 / 2} \frac{d r^{2}}{f(r)} \quad f(r)=1-\left(\frac{r_{k k}}{r}\right)^{3}
$$

where $\phi$ is periodic variable. Metric at low temperature is close to the thermal $A d S_{2}$. We insert D8-branes extended in radial coordinate $\mathrm{r}$ and localized at $\phi$ and D0 branes localized in radial coordinate in this background geometry and extended along $\phi$. The $N_{f} D 8-\bar{D} 8$ branes are connected at the tip of the cigar and are placed at $\phi=0, \pi$ on the $\phi$ circle. The D8 branes carries $U\left(N_{F}\right)$ flavor gauge group at the worldvolume and matrix $U$ of the pseudoscalar mesons $\pi_{a}$ is defined in terms of holonomy of radial component of the flavor gauge field

$$
U=e^{i t_{a} \pi_{a}}=e^{\int A_{r} d r}
$$

Above the critical temperature the metric is identified with the AdS BH in $N=4$ theory [12] and the phase transition in QCD qualitatively corresponds to the Hawking-Page transition. At $T=T_{c}$ the $\phi$ and $t_{E}$ coordinates get interchanged.

It is worth also to comment on the origin of the mass term $\operatorname{TrMU}$ in the Chiral Lagrangian. It was argued in $[24,25]$ that it comes from the worldsheet instanton that is the open string with worldsheet $(r, \phi)$ coordinates which is stretched between left and right D8 branes and spans some area on the cigar. The mass comes from the Nambu-Goto string action 
while the factor $U$ comes from the interaction of the open string end with D8 brane. We are interested in the Dirac operator eigenvalues that is purely imaginary masses. They can be obtained if the $\theta$ term is taken into account which holographically corresponds to the holonomy of the RR 1-form field along KK circle [27]. With the proper value of $\theta$ we get purely imaginary masses.

Remark that random fields act on open string not only at its ends. Indeed the QCD vacuum is populated by instantons and antiinstantons which are extended in $\phi$. The position of D0 instanton along the radial coordinate is known to be related with its size $\sigma$. Therefore density $d(\sigma) \propto \sigma^{-5}$ gets mapped into the density of the instantons along the radial coordinate.The instantons and antiinstantons act as random forces acting on the string worldsheet. The density of the external forces is r-dependent and the strength of the force follows from the "instanton mass"- its action $\exp \left(-\frac{8 \pi}{g^{2}(r)}+i \theta\right)$.

\section{Critical regime}

\subsection{Diagnostics of the critical behavior}

In what follows we shall be interested in the spectral properties near the mobility edge $E_{m}$. There are several specific features intrinsic for this regime supporting the multifractal behavior.

- First, the level spacing distribution $P(s)$ is the key indicator of the localization/delocalization transition. It behaves as

$$
\begin{cases}P_{\text {deloc }}(s)=A s e^{-B s^{2}} & \text { delocalized phase(GOE) } \\ P_{l o c}(s)=e^{-\frac{s}{2 \chi}} & \text { localized phase }\end{cases}
$$

The parameter $\chi$ in the Poisson tail is the level compressibility defined as

$$
\chi=\frac{d}{d \bar{n}}<(n-\bar{n})^{2}>, \quad N \gg \bar{n} \gg 1
$$

- The second indicator is the spectral formfactor which develops a fractal behavior at the mobility edge which differs both from Wigner-Dyson and Poisson statistics [33]

$$
R(\lambda) \propto \lambda^{-1+\frac{D_{2}}{d}}
$$

where $D_{2}$ is the fractal dimension defined as

$$
\sum_{r, n}<\left|\Psi_{n}(r)\right|^{2 p} \delta\left(E-E_{n}\right)>\propto L^{-D_{p}(p-1)}
$$

in the volume $L^{d}, \mathrm{~d}$ is dimension of space. The level number variance behaves as $\Sigma_{c r i t} \propto \chi E$ in the multifractal case, where the level compressibility reads as

$$
\chi=\frac{d-D_{2}}{2 d}
$$

- There is also specific violation of the normalization sum rules which yields the proper behavior for the level number variance

$$
(1-\chi)=2 \int_{s>0}^{\infty}\left(1-R_{\infty}\left(s^{\prime}-s\right)\right) d s^{\prime}
$$

The expression above is written for $\beta=2$, where $\beta$ is parameter of the $\beta$-ensemble, in the limit $e^{\frac{1}{x}} \gg 1$. 


\subsection{Matrix models for localization transition}

Turn now for the specific matrix models used for the critical statistics and multifractality of eigenstates. There are several critical matrix models [29-31] describing the localization transition in 3D which are qualitatively unified in [28]. All of them works only nearby the mobility edge (see [32] for review). Let us summarize their main features

- The two-matrix model [29] with the following probability function

$$
P(H) \propto \exp \left(-\beta \operatorname{Tr} H^{2}-\beta b \operatorname{Tr}\left([\Omega, H][\Omega, H]^{\dagger}\right)\right.
$$

where $\mathrm{b}$ is parameter and $\Omega$ is the fixed unitary matrix $\Omega=\operatorname{diag}\left(\exp \left(\frac{2 \pi i k}{N}\right)\right)$. The critical regime in this model implies that unitary symmetry breaking parameter behaves as $b=\mu N^{2}$ at $N \rightarrow \infty$. The matrix models of such types appeared in the context of the non-singlet sectors in $c=1$ string theory [39-41]. However contrary to that case we have no integration over $\Omega$ here and one could imagine that a kind of saddle-point value is taken into account.

- The second one-matrix model involves the potential providing the weak confinement [30] of eigenvalues. Such type of model was used in the matrix model description of ChernSimons theory [37] and was solved in terms of the q-Hermite polynomials. Asymptotically potential behaves as

$$
V(x) \rightarrow c \log ^{2} x, \quad x \rightarrow \infty
$$

where $c$ - is some parameter and the probability measure in the matrix integral reads as

$$
P(H) \propto \exp (-\beta \operatorname{Tr} V(H))
$$

The similar critical model for the chiral ensembles has been considered in [42].

- The third model was suggested in [31] and involves the Gaussian ensemble with independent random entries $(i \geq j)$

$$
<H_{i j}>=0, \quad<\left(H_{i j}\right)^{2}>=\beta^{-1}\left[1+\frac{(i-j)^{2}}{B^{2}}\right]^{-1}
$$

where B is parameter of the model, for $B>>1$ it gets mapped into supersymmetric sigma model. This model also manifests the multifractal behavior [31] at the mobility edge

The spectral correlators for all three critical models are the same

$$
R(E, s)=<\rho(E) \rho(E+s)>=\delta(s)+Y(E, s)
$$

where at small $\mathrm{s}$

$$
Y(E, s) \propto \frac{\pi^{2} \eta^{2}}{4} \frac{\sin ^{2}(\pi s)}{\sinh ^{2}\left(\pi^{2} s \eta / 2\right)} \quad \beta=2
$$

The parameter $\eta$ is related with the parameters of the matrix ensembles $\eta=\frac{c}{\pi^{2}}=\mu$ if we assume $\eta\langle 1$. The spectral correlator in this regime is identical to the density-density correlator for the free fermion gas at finite temperature proportional to $\eta$. The parameter $\eta$ is related with the fractal dimension as $\eta=1-D_{2}$. Similarly at small $\eta$ regime the spectral compressibility reads as

$$
\chi=1+\int_{-\infty}^{+\infty} Y(E, s) d s
$$

and is consistent with the general relation

$$
\chi=\frac{d-D_{2}}{2 d}
$$


Let us remark that formulas above are valid only for small multifractality. At large s there is the power-tail which knows about the fractal dimension as well. According to our conjecture the small s regime corresponds the IR region near the horizon while the large $\mathrm{s}$ regime captures the information about the UV scale. Since we are dealing with a kind of anomaly phenomena the information about the fractal dimension can be captured both in UV and IR regions.

\section{Mobility edge and BH horizon. Field theory}

Let us present a few qualitative arguments supporting the identification of the near horizon region in the holographic dual and the mobility edge in the Dirac operator spectrum.

- At the critical metal-insulator transition in the Dirac operator spectrum one could expect the jump of the chiral conductivity in the thermal QCD. The corresponding Kubo-like formula for the correlator of the Moether currents generated left and right chiral rotations reads as

$$
i \int d x<J_{v}^{L}(x) J_{\mu}^{R}(0)>=-\frac{1}{4} \eta_{\mu \nu} F_{\pi}^{2}
$$

Comparison of this QCD low-energy theorem with the formulas in the transport phenomena provides the identification of the $F_{\pi}$ as the diffusion coefficient in the chiral matter [43]. Hence we could ask when the jump of chiral conductivity is expected in the holographic setup. To be as model independent as possible consider the anomaly matching Son-Yamamoto condition in holographic QCD [34] which yields the relation between the 2- and 3-point functions and is diagonal with respect to the holographic RG flows [35]. The Son-Yamamoto relation amounts to the following expression for the "'running"' $F_{\pi}$

$$
F_{\pi}^{-2}(z)=\int_{0}^{z} \frac{1}{f^{2}(z)}
$$

which is valid for any holographic metrics. Taking the derivative of this expression we see immediately that the criticality for the conductivity takes place exactly at the $\mathrm{BH}$ horizon when $f(z)=0$.

- The lattice QCD studies demonstrate that the positions of mobility edge $\lambda_{m}(T)$ at $T>T_{c}$ grows as the function of the temperature near the deconfinement phase transition approximately as $\sqrt{T-T_{c}}$ [7]. This behavior qualitatively agrees with the holographic picture upon the proper identification of the $\lambda=0$ point in the radial coordinate in the BH geometry. To justify this identification it is suitable to use the Casher-Banks relation which relates the spectral density at the origin with the chiral condensate. The chiral condensate is identified with the tachyonic mode in the open string connecting the left and right D8 branes near the tip of the cigar [26]. Hence $\lambda=0$ is naturally identified with the position of the tip of the cigar. Nearby the transition point we could inspect the relation between the horizon scale and the temperature for AdS BH

$$
f\left(r_{h}\right)=0 \quad f(r)=1+r^{2}-\frac{M_{B H}}{r^{2}} \quad \beta=\frac{4 \pi}{f^{\prime}\left(r_{h}\right)}
$$

hence near the maximum of the function $T^{-1}\left(r_{h}\right)$ the relation holds

$$
\left(T-T_{c}\right) \propto\left(r-r_{h}\right)^{2}
$$

This behavior is qualitatively consistent with the observed temperature dependence of the mobility edge if we assume that $\lambda_{m}(T) \propto\left(r-r_{h}\right)$. 
Let us emphasize that the precise holographic metric in the deconfined QCD is unknown however the presence of horizon is well established. The detailed lattice study of the dependence $\lambda_{m}(T)$ could provide some information concerning the holographic metric.

- Recently the interesting relation for the spectral correlator has been found [36]. The analogue of the Casher-Banks relation for the spectral formfactor reads as follows

$$
R(\lambda=0, \lambda=0)-R_{\text {Pois }}(\lambda=0, \lambda=0)=f_{A}
$$

where $R\left(\lambda_{1}, \lambda_{2}\right)$-is the spectral correlator of the Dirac operator in the deconfined phase and $f_{A}$ is defined as the coefficient in front of $U(1)_{A}$ symmetry breaking term in the expansion of the partition function in terms of the fermion mass matrix $M$

$$
Z(M)=\exp \left(-\frac{V_{3}}{T}\left(f_{0}-f_{2} \operatorname{Tr} M^{+} M-f_{A}\left(\operatorname{det} M+\operatorname{det} M^{+}\right)+O\left(M^{4}\right)\right)\right)
$$

The (33) measures the difference between the spectral correlator in QCD and spectral correlator in the case of Poisson statistics. We know from the lattice studies that near the $\lambda=0$ the statistics is Poisson hence this observation implies $f_{A}=0$ and unbroken $U(1)_{A}$ symmetry. This issue is quite controversial and there are lattice results contradicting and supporting this statement in the literature. This argument certainly deserves for the additional study.

\section{Mobility edge and $\mathrm{BH}$ horizon. Worldsheet perspective}

The quark in the holographic representation corresponds to the open string connecting the D8 brane placed at $m_{q}$ scale and the IR region. In the confinement phase the string is extended along the holographic coordinate to the IR boundary and the tension of the string remains finite at all values of the radial coordinate. In the deconfined phase the string is embedded in the target differently. If a eigenvalue of the Dirac operator is large enough the main part of open string is extended along the radial coordinate towards the horizon while the second part of string lies at the near-horizon region where it is almost tensionless. These two parts of open string according to our conjecture correspond to the delocalized and localized eigenvalues of the Dirac operator correspondingly.

The arguments in the worldsheet theory go as follows. Since a eigenvalue of the Dirac operator corresponds to the imaginary quark mass the spectral properties of the quark wave function in the boundary theory get mapped into the worldsheet theory on the open string extended in the radial coordinate. For static quark the worldsheet of the open string involves the radial coordinate and the Euclidean time $t_{E}=\tau$. How the spectral statistics in the boundary theory gets translated into the worldsheet language? As we know the energy levels are independent in the localized phase and interact repulsively in the delocalized phase. The energy levels of the Dirac operator roughly correspond to bits of the open string. Using the spectrum - worldsheet correspondence we should analyze if the neighbor " "string bits" are correlated or independent. In the confined phase the string has the finite tension at all distances therefore tensionful string does not have any reason for uncorrelated neighbor bits. It is consistent with the Wigner-Dyson level statistics observed in lattice QCD in confined phase. In the deconfined phase the part of the string near horizon is almost tensionless and we conjecture that just this part of the string enjoys the Poisson statistics of the string bits.

To discuss the criticality from the worldsheet theory viewpoint note that the spectral correlator at the boundary gets mapped into correlator of densities of eigenvalues in the matrix model description of the string worldsheet theory

$$
<\rho(E) \rho\left(E^{\prime}\right)>_{R M T} \rightarrow<\rho(x) \rho\left(x^{\prime}\right)>_{\text {worldsheet }}
$$


in the thermodynamical limit. Similar mapping is known for a while [44] however in the holographic setting it acquires the very clear origin since the energy scale gets mapped into the value of the radial coordinate. Moreover the bulk metric induces the metric on the worldsheet, in particular in the deconfined phase the $\mathrm{BH}$ horizon induces the horizon on the worldsheet. The temperatures of the bulk and worldsheet black holes coincide for the static quark which has been clarified in the context of the holographic interpretation of drug force [45].

To get more insight on the interpretation of the density operator $\rho(x)$ in the worldsheet theory remind that the Wigner-Dyson RMT is equivalent to the ground state of the Calogero model for the fermions in the harmonic confining potential [44] with the Hamiltonian

$$
H_{C a l}=\sum_{i=1}^{N} p_{i}^{2}+\beta / 2(\beta / 2-1) \sum_{i<j} \frac{1}{\left(x_{i}-x_{j}\right)^{2}}+\omega^{2} \sum_{i=1}^{N} x_{i}^{2}
$$

The collective field $\rho(x, \tau)$ for the Calogero model is described in terms of the twodimensional scalar field as follows

$$
\rho(x, \tau)=\rho_{0}+A \cos (2 \pi x+2 \Phi(x, \tau))
$$

Hence the density-density correlator can be expressed in terms of the conventional Green function of the scalar $\Phi(x, \tau)$ in $(1+1)$. This is the way how the boundary spectral correlator in the confined phase is reproduced in the worldsheet theory.

It turns out [46] that the RMT-Calogero correspondence at zero temperature gets generalized to the relation between the Calogero model at finite temperature and the critical matrix model [30]. Once again the spectral correlator in the critical RMT gets mapped into the density-density correlator in the Calogero model at finite temperature upon the identification deformation parameter $b$ in the matrix model as [46]

$$
2 b=\frac{\omega}{\sinh \left(\frac{\omega}{T}\right)} \quad 2 b+1=\frac{\omega \cosh \left(\frac{\omega}{T}\right)}{\sinh \left(\frac{\omega}{T}\right)}
$$

Let us remind that coordinates of particles $x_{i}$ in Calogero model are their radial holographic coordinates while $\tau$ is the Euclidean time identified with the angular coordinate at the hyperbolic plane. The density-density correlator is taken at the same time. The Calogero model is considered at the fixed coupling constant which corresponds to the fermions.

Note that we can also use the duality found in [47] to map the rational Calogero model with the confining potential to the Sutherland model with the trigonometric interaction using the polar decomposition

$$
H_{S u t h}=\sum_{i=1}^{N} p_{i}^{2}+\beta / 2(\beta / 2-1) \sum_{i<j} \frac{1}{\sin ^{2}\left(x_{i}-x_{j}\right)}
$$

The time coordinate in Calogero gets mapped into the space coordinate in the Sutherland model. Both have interpretation of the Euclidean time in 4D Euclidean gauge theory. From the viewpoint of the Calogero model the radius of the Euclidean time coordinate corresponds to the inverse temperature while in the Sutherland model it yields the radius of the circle where the particles live. The density-density correlator in the Calogero model gets mapped into more complicated "'mixed"' correlator in the trigonometric Sutherland model.

The Sutherland representation of spectral correlator provides one more link with the disorder picture. It was found in [48] that the Sutherland model at the boundary of the hyperbolic plane has the interpretation in terms the multiple interacting radial SLE stochastic process. 
The strings are extended from the boundary into the bulk and are affected by the random force. If string ends in the bulk obey the SLE dynamics the boundary degrees of freedom evolve under the Sutherland Hamiltonian. In our worldsheet picture it means that we have large number of strings extended into radial direction in the random environment which are attached to the vortices on the string worldsheet.

Another way to get the same critical behavior is to consider the scalar field not on the thermal cylinder but in the $2 \mathrm{~d}$ curved background. It was observed in [49] that the background can be curiously identified with the effective $2 \mathrm{~d}$ "'acoustic $\mathrm{BH}$ "'. In fact the only important point we need is that the background provides the horizon and effective Hawking temperature. This viewpoint reminds logic of our study when the $2 \mathrm{~d} \mathrm{BH}$ metrics on the worldsheet is induced from the bulk involving $\mathrm{BH}$. The fractal dimension $D_{2}$ derived in [49] is related to the Hawking effective temperature $\mathrm{T}$ as

$$
T \propto \pi \frac{d-D_{2}}{d}
$$

To get more insight on the interpretation of collective field $\Phi(s, \tau)$ remind that the $\cos \Phi$ terms appeared in the description of the string in the $2 \mathrm{~d} \mathrm{BH}$ background [38]. They came from the modes with the unit winding around the compact dimension. It was argued [38] that the condensation of winding modes takes place near the BH horizon. Hence we could suggest that in our study the scalar field has the same meaning as the collective field for the vortices. The vortices come from the non-singlet sector in the $c=1$ matrix model [39-41]. It is these vortices which yield the Calogero model and the Luttinger effective collective field description.

Inspiring remark concerns the semiclassical expression for the spectral formfactor $K(t)$ in terms of the return probability $p(t)[50]$ in the non-relativistic limit

$$
K(t) \propto|t| p(t)
$$

When $t$ is the usual time the return probability has the evident interpretation in terms of the space-time trajectories. In that case (41) relates the transport properties connected with the return probability and the spectral properties of the system. However in our study the " time"' variable has the meaning of the RG scale hence the formula like (41) has to be reinterpreted. Naively the return probability in the RG flows has the meaning of the cyclic RG flow which occurs in several examples involving two charge RG flows. It would be very interesting to recognize if the cyclic RG flows are important for the spectral formfactor in the worldsheet description. Remark that in our study we deal with the relativistic fermion in 4 dimensions hence (41) has to be generalized.

It is worth to mention the relation of our long string arguments with two other problems. First, it was suggested in [51] that the deconfinement phase transition is related to the condensation of the long strings forming the horizon. Another related problem concerns the holographic interpretation of the entropic forces relevant for the meson dissociation [52] in the deconfined phase. The string connecting the quark and antiquark has a fragment lying at the horizon radial coordinate and it was argued that just this fragment provides the large coordinate dependent entropy and therefore the entropic force. It looks that the fragment at the horizon radial scale is effectively broken into the independent bits. This is somewhat parallel to our case hence we could claim that the string bits at the horizon in the dissociation problem enjoy the Poisson statistics. The potential mechanism which would relate the Poissson statistics and the dissociation phenomena is the formations of the multiple hole in the string worldsheet in the near horizon limit. In the Euclidean regime it would correespond to the intependence of the neighbor string bits while upon the continuation to the Minkowski space it will corresponds to the decay of the confining string into the muptiple fragments. 


\section{Conclusion}

Our study provides some evidences that the mobility edge in the Euclidean 4D Dirac operator spectrum in the deconfined phase of QCD corresponds to the BH near horizon region in the holographic dual however further clarification is certainly required. It is natural to assume that this correspondence is quite general phenomenon and in particular the $2 \mathrm{~d} \mathrm{BH}$ in the dilaton JT gravity could be dual to SYK model [53](see [54] for review and references) only if we deform it to provide the criticality in its spectrum. The precise example of the emerging mobility edge in the perturbed SYK has been found recently in [55]. Hence it is natural to conjecture that the mobility edge in the properly perturbed SYK corresponds to the $2 \mathrm{D} \mathrm{BH}$ near horizon region.

It would be also interesting to match our study with the discussion in [56]. It was argued there that quite generically criticality appears in the ungapped phase of boundary Euclidean theory at the horizon and its origin is the lack of possibility to match the initial UV state with the particular state in IR through the holographic RG flow. The tensor network language has been used there to clarify this statement. Our study suggests that the corresponding criticality at horizon is expected to be the metal-insulator type transition in the spectrum of the boundary Euclidean theory.

Another important point suggested by our study is that it would be very interesting to investigate in detail in the lattice QCD framework the low-energy eigenmodes of the Dirac operator in the deconfined QCD. It would provide the interesting information concerning the near-horizon region of the $\mathrm{BH}$ in $5 \mathrm{D}$. The very confinement phenomena corresponds to the decay of the $\mathrm{BH}$ horizon into thermal gravitons. It would be also interesting to investigate the case of deconfinement transition induced by baryonic density from the viewpoint of Dirac operator spectrum.

In [57] the interesting relation between the condensate and torus knot invariants has been found. It turned out that a fermion condensate in $S U(2)$ SUSY QCD plays the role of generating function for the torus knot invariants. Torus knots are placed in the internal "'momentum"' space however they are intimately related with the invariants of the moduli space of instantons sitting at one point at the top of each other in the physical Euclidean 4D spacetime. It would be interesting to question if similar relation between the knot invariants and the Dirac operator spectrum in QCD exists in the instanton-antiinstanton vacuum ensemble.

I am grateful to M.Litvinov and N.Sopenko for collaboration and to V. Braguta, A. Kamenev, D. Kharzeev, V. Kravtsov, A. Milekhin, S. Nechaev, N. Prokof'ev , B. Shklovskii for the useful discussions.

\section{References}

[1] A. Gorsky, M. Litvinov and N. Sopenko, "'Metal or insulator? Dirac operator spectrum in holographic QCD"', to appear

[2] T. Banks and A. Casher, Nucl. Phys. B 169, 103 (1980).

[3] H. Leutwyler and A. V. Smilga, Phys. Rev. D 46, 5607 (1992).

[4] J. J. M. Verbaarschot and T. Wettig, Ann. Rev. Nucl. Part. Sci. 50, 343 (2000) [arXiv:hepph/0003017], R. A. Janik, M. A. Nowak, G. Papp and I. Zahed, Acta Phys. Polon. B 29, 3957 (1998) [hep-ph/9812376], G. Akemann, arXiv:1603.06011 [math-ph].

[5] J. Stern, [hep-ph/9801282], R. A. Janik, M. A. Nowak, G. Papp and I. Zahed, Phys. Rev. Lett. 81, 264 (1998), J.C. Osborn and J.J.M. Verbaarschot, Phys. Rev. Lett. 81 (1998) 268, Nucl.Phys. B 525 (1998) 738

[6] A. M. Garcia-Garcia and J. C. Osborn, Nucl. Phys. A770, 141 (2006),

A. M. Garcia-Garcia and J. C. Osborn, Phys. Rev. D 75,034503 (2007) 
[7] M. Giordano, T. G. Kovacz and F. Pittler, Phys. Rev. Lett., 112, no. 10, 102002 (2014),

T. G. Kovacs and R. A. Vig, arXiv:1706.03562 [hep-lat].

[8] R. Gopakumar, Phys. Rev. D 70, 025009 (2004)

[9] A. Gorsky and V. Lysov, Nucl. Phys. B718, 293 (2005)

[10] K. Furuuchi, Phys. Rev. D 72, 066009 (2005)

[11] J. de Boer, E. P. Verlinde and H. L. Verlinde, JHEP0008, 003 (2000)

[12] E. Witten, Adv. Theor. Math. Phys. 2, 505 (1998) [hep-th/9803131].

[13] O. Aharony, J. Marsano, S. Minwalla, K. Papadodimas, and M. Van Raamsdonk, "Adv. Theor. Math. Phys.8 (2004) 603-696, arXiv:hep-th/0310285 [hep-th]

[14] E. Fradkin Phys. Rev. B 33, 3257 ,1986; Phys. Rev. B 33, 3263, 1986

[15] S.V. Syzranov, L. Radzihovsky Ann. Rev. Cond. Mat. Phys. 9, 35 (2018)

S.V. Syzranov, V. Gurarie, L. Radzihovsky Ann. Phys. 373, 694 (2016)

[16] P. Damgaard Phys.Lett.B424 (1998) 322, hepth/9807026

[17] G. Parisi, J. Phys. A 14 (1981) 735.

[18] T. Morimoto, A. Furusaki, C. Mudry Phys. Rev. B 91, 235111 (2015)

[19] A. Altland, D. Bagrets, A. Kamenev Phys. Rev. B 91, 085429 (2015)

[20] D. Khmelnitskii, JETP Lett 38 (552, (1983)

[21] A. Pruisken Nucl.Phys. B 235, 277 (1984)

[22] D. Gaiotto, A. Kapustin, Z. Komargodski and N. Seiberg, JHEP 1705, 091 (2017) [arXiv:1703.00501 [hep-th]].

[23] T. Sakai and S. Sugimoto, Prog. Theor. Phys. 113, 843 (2005) [hep-th/0412141].

[24] O. Aharony and D. Kutasov, Phys. Rev. D 78, 026005 (2008) [arXiv:0803.3547 [hepth]].

[25] K. Hashimoto, T. Hirayama, F. L. Lin and H. U. Yee, JHEP 0807, 089 (2008) [arXiv:0803.4192 [hep-th]].

[26] O. Aharony, J. Sonnenschein and S. Yankielowicz, Annals Phys. 322, 1420 (2007) [hepth/0604161].

[27] E. Witten, Phys. Rev. Lett. 81, 2862 (1998) [hep-th/9807109].

[28] V.E.Kravtsov, K.A.Muttalib Phys.Rev.Lett., 79 (1997) 1913.

[29] A. Mirlin et al Phys.Rev.E 54, 3221 (1996)

[30] K. Muttalib et al PRL 71, 471,(1993)

[31] M. Moshe,, H.Neuberger and B. Shapiro PRL 73, 1497 (1994)

[32] V.E.Kravtsov "'Random matrix representations of critical statistics"' , in the Handbook on Random Matrix Theory , arXiv:0911.0615

[33] V. E. Kravtsov, I. V. Lerner, B. L. Altshuler, A. G. Aronov PRL, vol. 72, p.888 (1994) cond-mat/9402026.

V.E.Kravtsov ,Spectral Statistics at the Anderson Transition: Multifractality of Wave Functions and the Violation of the Normalization Sum Rule arXiv:cond-mat/9603166

J. T. Chalker, V. E. Kravtsov, I. V. Lerner JETP Lett. 64 (1996) 386

[34] D. T. Son and N. Yamamoto, arXiv:1010.0718 [hep-ph]

[35] O. Dubinkin, A. Gorsky and A. Milekhin, Phys. Rev. D 91, 066007 (2015) [arXiv:1412.0513 [hep-th]].

[36] T. Kanazawa and N. Yamamoto, JHEP 1601, 141 (2016) [arXiv:1508.02416 [hep-th]].

T. Kanazawa, JHEP 1510, 010 (2015) [arXiv:1507.06376 [hep-ph]].

[37] M. Tierz Mod.Phys.Lett.A24:3157-3171,2009

R. J. Szabo and M. Tierz, J. Phys. A 43, 265401 (2010) [arXiv:1003.1228 [hep-th]]. 
[38] V. Kazakov, I. K. Kostov, and D. Kutasov, Nucl. Phys.B622 (2002) 141-188, arXiv:hepth/0101011 [hep-th]

[39] D. J. Gross and I. R. Klebanov, Nucl. Phys. B354 (1991) 459-474.

[40] D. Boulatov and V. Kazakov, Int. J. Mod. Phys. A 8, 809 (1993) [hep-th/0012228].

[41] J. M. Maldacena, ’JHEP, 09(2005) 078, arXiv:hep-th/0503112 [hep-th]

J. Maldacena and A. Milekhin, JHEP 1804, 084 (2018)

[42] A. M. Garcia-Garcia and J. J. M. Verbaarschot, Nucl. Phys. B 586, 668 (2000) [hepth/0003159].

[43] R. A. Janik, M. A. Nowak, G. Papp and I. Zahed, Phys. Lett. B 442, 300 (1998) [hep$\mathrm{ph} / 9807550]$.

P.H. Damgaard, U.M. Heller, R. Niclasen and K. Rummukainen Low-lying Eigenvalues of the QCD Dirac Operator at Finite Temperature hep-lat/0003021.

F. Farchioni, P. de Forcrand, I. Hip, C.B. Lang, and K. Sp littorff, hep-lat/9912004

J.J.M. Verbaarschot, Randomness on the Lattice arXiv:hep-lat/0008025

[44] B. D. Simons, P. A. Lee and B. L. Altshuler, Nucl. Phys. B 409, 487 (1993).

[45] S. S. Gubser, Phys. Rev. D 76, 126003 (2007) [hep-th/0611272].

[46] A. M. Garcia-Garcia and J. J. M. Verbaarschot, Phys. Rev. E67, 046104 (2003),

[47] N. Nekrasov, "On a duality in Calogero-Moser-Sutherland systems," hep-th/9707111.

[48] J. L. Cardy, Phys. Lett. B 582, 121 (2004) [hep-th/0310291].

[49] F. Franchini, V. E. Kravtsov Phys. Rev. Lett. 103: 166401,2009

[50] N.Argaman, Y. Imry and U. Smilyansky, Phys. Rev B(1993),

[51] M. Hanada, J. Maltz and L. Susskind, Phys. Rev. D 90, no. 10, 105019 (2014)

[52] K. Hashimoto and D. E. Kharzeev, Phys. Rev. D 90, no. 12, 125012 (2014) [arXiv:1411.0618 [hep-th]].

[53] S. Sachdev and J. Ye, Phys. Rev. Lett. 70, 3339 (1993).

A.Kitaev,"A simple model of quantum holography," KITP strings seminar and Entanglement 2015 program, 12 February,7 April and 27 May 2015,

[54] V. Rosenhaus, "An introduction to the SYK model," arXiv:1807.03334 [hep-th].

[55] A. M. García-García, B. Loureiro, A. Romero-Bermúdez and M. Tezuka, Phys. Rev.

Lett. 120, no. 24, 241603 (2018) [arXiv:1707.02197 [hep-th]].

[56] S. S. Lee, JHEP 1609, 044 (2016) [arXiv:1603.08509 [hep-th]].

[57] A. Gorsky, A. Milekhin and N. Sopenko, JHEP 1509, 102 (2015)

A. Gorsky and A. Milekhin, Nucl. Phys. B 900, 366 (2015) 